\title{
Evidence of low-density water to high-density water structural transformation in milk during high- pressure processing
}

\author{
Bérengère Guignon a, b, ${ }^{*}$, Eduardo Hidalgo Baltasar a, Pedro D. Sanz ${ }^{b}$, \\ Valentín G. Baonza a , Mercedes Taravillo ${ }^{a}$
}

a MALTA Consolider TEAM, Departamento de Química Física I, Facultad de Ciencias Químicas, UCM, Avda. Complutense s/n, 28040-Madrid, Spain.

b MALTA Consolider TEAM, Departamento de Procesos, Instituto de Ciencia y Tecnología de Alimentos y Nutrición, CSIC, C/ José Antonio Nováis 10, 28040Madrid, Spain.

${ }^{*}$ Corresponding author.

E-mail addresses: bguignon@ucm.es (BG); eduardohidalgobaltasar@gmail.com (EHB); pedro.sanz@csic.es (PDS); vgbaonza@quim.ucm.es (VGB); mtaravil@quim.ucm.es (MT).

\begin{abstract}
The objective of this research was to check whether the observed low-density water (LDW) to high-density water (HDW) transformation does take place or not in a complex aqueous system like those involved in high pressure processing of food. In-situ measurements of speed-of-sound up to $640 \mathrm{MPa}$ were used for this purpose. After validation of the methodology in liquid water at $25^{\circ} \mathrm{C}$, LDW-to-HDW transformation was also evidenced in sodium caseinate solution and milk samples. The transformation pressure was always observed at $275 \mathrm{MPa}$. Since water plays a key role in most biochemical transformations, the occurrence of a LDW-HDW transition should be taken into account for understanding the complex component interactions in milk and other related systems under pressure.

Industrial relevance: Opportunities exist for the industry to use pressure as a tool for texturing dairy products. Process parameter choice to obtain a given texture is tricky due to the complexity of milk component interactions under pressure. As a main component in foods, water structural transformation under pressure should not be ignored by experts in the field.
\end{abstract}

Keywords: High hydrostatic pressure process; High-density water; Ultrasounds; Milk; Sodium caseinate; Hydrogen bond.

\section{Introduction}

High-pressure processing of foods continues extending in the industry as a mild preservation treatment. Pressure effects are also interesting for texturing dairy products (Devi, Buckow, Hemar, \& Kasapis, 2013). In spite of a great deal of researches in this field, food texture modulation by pressure treatment is still challenging. The mechanisms leading to a given texture observed after treatment are not fully understood.

In-situ techniques are useful to elucidate these mechanisms (Tromp, Huppertz, \& Kohlbrecher, 2014; Gebhardt, Takeda, Kulozik, \& Doster, 2011). Here, we introduce 
the use of ultrasonic measurements to address pressure-induced phenomena during high pressure processing of milk. In particular, we are going to focus on pressure-induced changes in water.

Liquid water has a short-range molecular structure. This structure emerges from the dynamic network formed by the hydrogen bonds between water molecules. It can be roughly considered that a given water molecule has four first neighbors arranged in a quasi-tetrahedral way around it (first shell), and other four neighbors at a higher distance (second shell). When pressure is increased, the second shell collapses into the first one and the hydrogen bonding network is strongly modified (Okhulkov, Demianets, \& Gorbaty, 1994). As a result, a pressure-driven structural rearrangement of water takes place in the liquid phase. This is known in the specialized literature as the Low-Density Water (LDW) to High-Density Water (HDW) structural transformation (Soper \& Ricci, 2000). It was shown by both calculations and experiments that water exhibits such transformation between $200 \mathrm{MPa}$ and $300 \mathrm{MPa}$ at room temperature (Fanetti et al., 2014; Li et al., 2005; Marco Saitta \& Datchi, 2003).

Since these pressure-temperature conditions are commonly achieved during high pressure processing of food, the question that arises is whether such structural transformation also happens in more complex aqueous systems (e.g. milk). This is an important question because it has long been suspected that water gets involved in protein denaturation and changes in casein micelles under pressure (Huppertz \& de Kruif, 2006), and both phenomena have an impact on dairy product texture; however, water implication in such phenomena has not been strictly demonstrated yet.

Here our goal is to seek for evidences of such structural transformation in milk under pressure. For this purpose, we first demonstrate how LDW-to-HDW transformation can be detected in pure water by ultrasound measurements, and later, we shall check whether it is observed or not in two selected complex systems of interest in food industry: sodium caseinate solution and pasteurized skimmed milk.

\section{Material and methods}

\subsection{Samples}

Deionized water type I (electrolytic conductivity $\sim 0.05 \mu \mathrm{S} \cdot \mathrm{cm}^{-1}$, Milli-Q system, Millipore, Billerica, USA) was employed in the experiments.

Sodium caseinate solution was chosen to start the study in complex systems because it is comparatively less complex than milk and because of its importance in the dairy sector for cheese making and as an additive in foods. The aqueous solution of sodium caseinate (Ref. C8654 - from bovine milk, batch no $117 \mathrm{~K} 0138$, Sigma-Aldrich, New Zealand) was prepared at a mass fraction of 0.026 which is the mean casein concentration in milk. Moisture content of sodium caseinate was taken into account ( $6.2 \%$ dry basis from thermogravimetric analysis). The caseinate powder was dissolved in deionized water by continuous stirring at room temperature the day before the experiments.

The study was then pursued with milk (Puleva, local supermarket, Spain). Milk was chosen skimmed and pasteurized to minimize the potential interferences of pressure-induced phenomena other than water transformation (e.g. fat crystallization, casein micelles disruption and whey protein denaturation) during the measurements (Harte, Gurram, Luedecke, Swanson, \& Barbosa-Cánovas, 2007). The claimed composition was $4.7 \mathrm{~g}$ of carbohydrates, $3.1 \mathrm{~g}$ of proteins, $0.3 \mathrm{~g}$ of fat, and $110 \mathrm{mg}$ of calcium for $100 \mathrm{~mL}$ of milk.

\subsection{Ultrasonic measurements}


The speed of sound in the samples was measured in situ as a function of pressure employing the same experimental setup as described in a previous work (Hidalgo Baltasar, Taravillo, Baonza, Sanz, \& Guignon, 2011). This setup has the particularity to cover the intermediate pressure range between (100 and 700) $\mathrm{MPa}$ in comparison to commercial devices (usually limited to $300 \mathrm{MPa}$ ) and diamond anvil cell (usually used above $1000 \mathrm{MPa}$ ).

The experimental procedure for measurements consists of: sample holder filling (about $15 \mathrm{~mL}$ of sample), ultrasonic cell setting up, vessel load and closing, thermal equilibration, pressure increase step by step (either 10 or $20 \mathrm{MPa}$ steps), and final decompression. At each step, pressure, temperature, and ultrasound signal are simultaneously recorded. The maximal combined standard uncertainties are estimated to be $0.2^{\circ} \mathrm{C}$ in temperature and $3.2 \mathrm{MPa}$ in pressure. Speed of sound is calculated from the ratio between the wave travel path and the time of flight. Both parameters are obtained exactly in the same way as explained in detail earlier (Hidalgo Baltasar et al., 2011). Experiments were repeated three times. The combined standard uncertainty in speed of sound is estimated to be between (2 and 4) $\mathrm{m} \cdot \mathrm{s}^{-1}$.

\subsection{Data treatment}

Numerical analyses of the results (signal analysis, non-linear curve fitting) were performed using OriginPro 8.0 package (OriginLab Corporation, Northampton, MA, USA).

\section{Results and Discussion}

\subsection{Determination of HDW-to-LDW transformation by ultrasound measurements}

The study of pure liquid water is the first step and a key step in the understanding of most food systems submitted to different temperature and pressure conditions. This is still also one of the biggest challenges to scientists and technologists since it is well known that many of its static and dynamic properties present an anomalous behavior. Among the anomalies found in the structural properties of water at the molecular level, the LWD-to-HDW transformation is one of the most intriguing. Despite it has been studied by different techniques (Fanetti et al., 2014; Li et al., 2005; Marco Saitta \& Datchi, 2003), the knowledge about this transformation remains incomplete. Here we study it by means of acoustic measurements since the speed of sound gives simultaneous information on several thermodynamic properties (specific heat, thermal expansion coefficient) and, in particular, on density (Guignon \& Baonza, 2016).

Speed of sound was determined in water as a function of pressure at $25^{\circ} \mathrm{C}$. The results are shown in Figure 1. Speed of sound in water increases with pressure in a non-linear way. Because the liquid becomes denser, the ultrasonic wave is transmitted faster under pressure. An excellent agreement is found with reference data: fractional deviations from IAPWS-95 formulation are below $0.5 \%$.

Speed of sound is related to density $\rho$ and adiabatic compressibility $\kappa_{s}$ through the Newton-Laplace equation:

$$
w=\sqrt{\frac{1}{\kappa_{s} \cdot \rho}}
$$

And by definition:

$$
\kappa_{S}=\frac{1}{\rho}\left(\frac{\partial \rho}{\partial p}\right)_{S}
$$




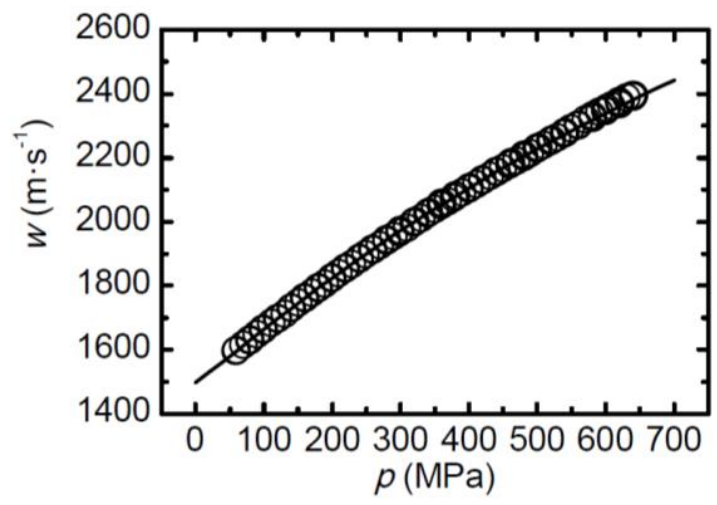

Figure 1. Measured speed-of-sound in water as a function of pressure compared to IAPWS-95 formulation (solid line, water equation-of-state from Wagner \& Pruss, 2002).

Therefore, we can easily compute density change with pressure from the speed-ofsound measurements:

$$
\left(\frac{\partial \rho}{\partial p}\right)_{s}=\frac{1}{w^{2}}
$$

This is plotted in Figure $2 \mathrm{a}$ in density unit $\left(\mathrm{s}^{2} \cdot \mathrm{m}^{-2}=10^{6} \cdot \mathrm{kg} \cdot \mathrm{m}^{-3} \cdot \mathrm{MPa}^{-1}\right)$. The rate at which density increases with pressure continuously decreases also in a non-linear way.

(a)

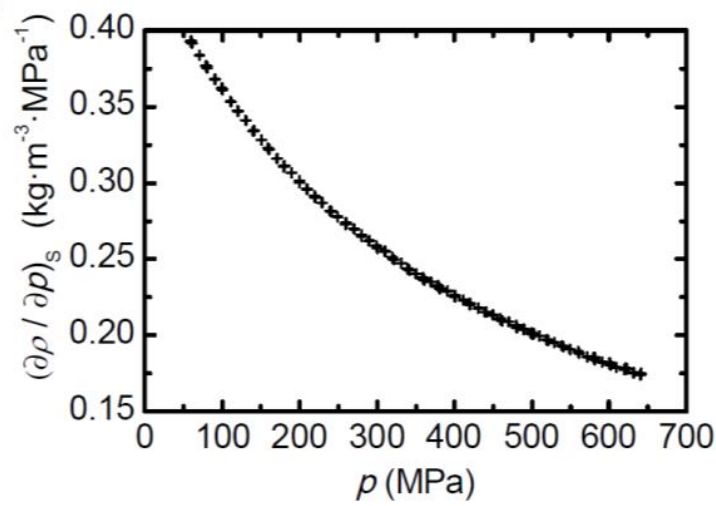

(b)

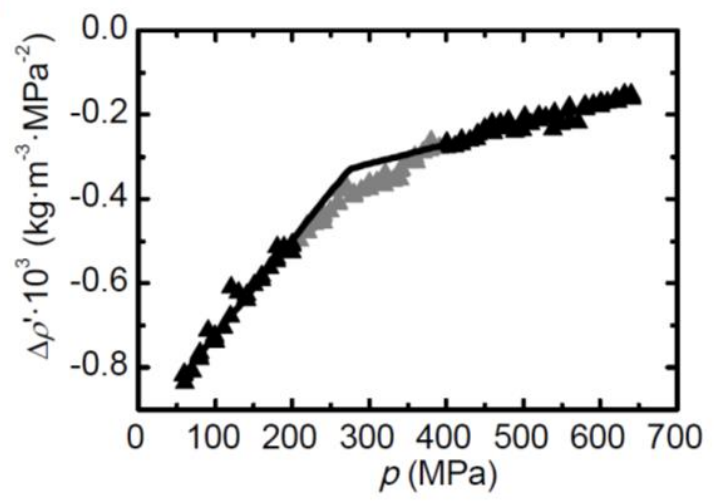

Figure 2. Water density variation (a) and its numerical derivative (b) as functions of pressure. The two lines are the results of fitting to two straight lines intersecting at the LDW-HDW transformation pressure, see Equation (5). Grey points deviate from the limiting linear behavior due to the changes in HDW and LDW fractions; so they were disregarded from the fit.

The pressure dependencies of both $w$ and $1 / w^{2}$ are smooth and there are no obvious changes with pressure. However, if we evaluate the pressure derivative of $1 / w^{2}$, two asymptotic linear trends are observed (Figure 2b). The derivative is numerically computed as follows: 


$$
\Delta \rho^{\prime}\left(p_{i}\right)=\frac{1}{2}\left(\frac{\rho_{i+1}^{\prime}-\rho_{i}^{\prime}}{p_{i+1}-p_{i}}+\frac{\rho_{i}^{\prime}-\rho_{i-1}^{\prime}}{p_{i}-p_{i-1}}\right) \text { with } \rho_{i}^{\prime}=\left(\frac{\partial \rho}{\partial p}\right)_{S} \text { at } p=p_{i}
$$

Although this differential expression unavoidably leads to an increase of uncertainty, the low experimental uncertainty of the raw data and the small pressure derivation step used in calculations still allow obtaining a semi-quantitative picture of the studied phenomena. The uncertainty in $\Delta \rho^{\prime}$ is estimated to be below $4 \cdot 10^{-5} \mathrm{~kg} \cdot \mathrm{m}^{-}$ 3. $\mathrm{MPa}^{-2}$ (law of propagation of uncertainty).

The characteristic slopes of the linear parts of the computed derivative $\Delta \rho^{\prime}(p)$ are listed in Table 1 . These slopes, $C_{1}$ and $C_{2}$, as well as the pressure of intersection of the lines, $p_{\text {trans, }}$ were obtained by non-linear curve fitting (Levenberg-Marquardt algorithm) according to the following model:

$$
\begin{aligned}
& \text { If } p<p_{\text {trans }}: \\
& \quad \Delta \rho^{\prime}(p)=C_{1} \cdot p+p_{\text {trans }}\left(C_{2}-C_{1}\right)+C_{3} \\
& \text { else : } \\
& \quad \Delta \rho^{\prime}(p)=C_{2} \cdot p+C_{3}
\end{aligned}
$$

$p_{\text {trans, }} C_{1}, C_{2}$ and $C_{3}$ are the fitted coefficients.

The two linear parts reflect two different water density behaviors attributable to a LDW structure at pressures below $200 \mathrm{MPa}$ and to a HDW structure at pressure above $400 \mathrm{MPa}$. The transformation between both structures is not sharp but rather occurs over a range of pressures as suggested by several authors (Soper and Ricci, 2000; Koga, Westh, Yoshida, Inaba, \& Nakazawa, 2014). Therefore, before fitting, the central points between (200 and 400) MPa which correspond to the transformation region were discarded. If all data points would be included, $p_{\text {trans }}$ would be upshifted in about $7 \mathrm{MPa}$ to $14 \mathrm{MPa}$ (parameters not shown). By excluding the central points from the fitting, we are slightly improving the parameter determination since the linear behavior of each main phase is privileged. The parameter $p_{\text {trans }}$ should be interpreted as the central point of the pressure interval over which the LDW-to-HDW transformation occurs.

Three sets of experimental data are used in the fitting procedure (superimposed data in Figure 2b). Thank to this, the fitting algorithm provides a relevant estimate of the standard uncertainty in the fitted parameter (figures shown next to the parameters in Table 1). The uncertainty in the determination of $p_{\text {trans }}(6 \mathrm{MPa})$ is higher than the experimental uncertainty in pressure (1.4 MPa). This is the expected consequence of the numerical derivation. However, this uncertainty still remains small and is relevant considering the pressure range of interest in food processing.

\begin{tabular}{ccccc}
\hline & $\frac{p_{\text {trans }}}{\mathrm{MPa}}$ & $\frac{\mathrm{C}_{1} \cdot 10^{6}}{\mathrm{~kg} \cdot \mathrm{m}^{-3} \cdot \mathrm{MPa}^{-3}}$ & $\frac{\mathrm{C}_{2} \cdot 10^{6}}{\mathrm{~kg} \cdot \mathrm{m}^{-3} \cdot \mathrm{MPa}^{-3}}$ & $\frac{\mathrm{C}_{3} \cdot 10^{4}}{\mathrm{~kg} \cdot \mathrm{m}^{-3} \cdot \mathrm{MPa}^{-2}}$ \\
\hline Water & $274 \pm 6$ & $2.27 \pm 0.06$ & $0.47 \pm 0.03$ & $-4.57 \pm 0.14$ \\
$\begin{array}{c}\text { Sodium caseinate } \\
\text { solution } \\
\begin{array}{c}\text { Pasteurized } \\
\text { skimmed milk }\end{array}\end{array}$ & $275 \pm 3$ & $2.21 \pm 0.03$ & $0.49 \pm 0.01$ & $-4.55 \pm 0.06$ \\
\hline
\end{tabular}

Table 1. Fitted coefficients of Equation (5) obtained from data of Figure $2 b$ for water and Figure 3 for the indicated systems. 
Our results evidence the two different forms of liquid water, LDW and HDW, with a transformation pressure ( $p_{\text {trans }}$ ) between both, located at $(274 \pm 6) \mathrm{MPa}$. This transformation pressure found here at room temperature is in agreement with previous works (Marco Saitta \& Datchi, 2003; Li et al., 2005; Fanetti et al., 2014). As expected, the density of LDW varies larger and faster than that of HDW with increasing pressure.

With this approach, we have validated our methodology for the detection of HDW, but its interpretation require more or less elaborated structural models. Current structural models of water invoke the dynamic formation and disruption of local pentameric aggregates with geometries reflecting a fourfold $\mathrm{H}$-bond coordination relative to a central $\mathrm{H}_{2} \mathrm{O}$ molecule. And both molecular dynamics simulations (Marco Saitta \& Datchi, 2003) and radial distribution functions obtained in diffraction experiments (Okhulkov et al., 1994) suggest that liquid water itself can be seen as a random network of molecules with three-four hydrogen bonds on average. Many anomalies have been associated with different interactions between these pentameric units that give rise to the appearance of two minima in the interaction potential energy, and some authors attribute the LWD-to-HDW transformation to the existence of interstitial water molecules in the pentameric unit (Marco Saitta \& Datchi, 2003). However, the question of whether such a transition is sharp (first order) or continuous is still open. In a recent study (Weck et al., (2009)), it has been suggested that the structural evolution of water is continuous, with the oxygen coordination number going from 4 to 12 over a fairly small density range, and hence, not associated with a first-order transition from the LDW form to the HDW form of water. The structural changes have been also confirmed from the analysis of Raman spectroscopy experiments (Baonza, Rull, \& Dubessy, 2012). This is in excellent agreement with our observations, as the changes observed in the derivative of the density are also continuous, and justifies the numerical analysis described here.

\subsection{Evidence of LDW-to-HDW transformation in complex food systems}

The question is now to investigate whether this structural change in water also happens when complex solutes are present. Sodium caseinate in water $(0.026$ mass fraction) forms a colloidal suspension of small casein aggregates. Milk is also a colloidal system where the non-aqueous components amount to near $8 \% \mathrm{w} / \mathrm{v}$ of the composition. These components are mainly caseins and whey proteins, carbohydrates, lipids, vitamins, and mineral salts, distributed between the continuous and colloidal phases. The presence of these components alters neat water structure. Consequently, the LDW-to-HDW transformation could be shifted to a different pressure (by promoting or inhibiting it). As stated in introduction, this information is relevant to obtain a more complete picture of what occurs in milk under pressure. Water is the key actor of hydrophobic interactions. Thus, any change in the structure of liquid water should have an impact on hydrophobic effects and then could modify, for examples, protein-protein and protein-solvent interactions.

In order to establish if water structure still transforms under pressure in these systems, our methodology for the detection of LDW-to-HDW transformation was applied. The milk sample characteristics were selected so as to minimize the response to pressure of non-aqueous components. In this way, it is known that lactose is stable at $25^{\circ} \mathrm{C}$ below $700 \mathrm{MPa}$ (Moreno, Villamiel, \& Olano, 2003) and it is also known that heat-denatured whey proteins link to caseins and prevent micelles from pressure-induced size changes (Harte, Gurram, Luedecke, Swanson, \& Barbosa-Cánovas, 2007). Moreover, lipid content was reduced to avoid the potential contribution of fat crystallization caused by pressure to speed-of-sound variation. Therefore, the ultrasonic signature of water structural transformation cannot be masked by any other one.

Speeds of sound in sodium caseinate solution and in pasteurized skimmed milk were measured as a function of pressure at $25^{\circ} \mathrm{C}$ and the measurements were 
submitted to the same numerical treatment as described above for water. The results are shown in Figure 3 and Table 1. The numerical pressure derivative $\Delta \rho^{\prime}(p)$ displays the same change of trend at $(275 \pm 3) \mathrm{MPa}$ for sodium caseinate solution, and at $(277 \pm 4) \mathrm{MPa}$ for skimmed milk, as for water. Since non-aqueous components have here a negligible contribution to density variation with pressure, the observed changes of trend around $275 \mathrm{MPa}$ can only be ascribed to water. The slopes of the lines are also almost identical. This is undoubtedly the same transformation as observed for pure water. The so-called LDW-to-HDW structural transformation does take place also in presence of casein aggregates and other non-aqueous milk components.

Quite surprisingly, concentration does not appear to have any influence on the transformation pressure in the studied systems, at least up to $8 \% \mathrm{w} / \mathrm{v}$ total concentration. It is possible that the pressure shift might be smaller than the experimental uncertainty and thus cannot be detected, or that the size, type and aggregate state of solutes have their importance (no colligative effect) as already observed in the case of surfactant micelles (Hidalgo Baltasar, Taravillo, Sanz, Baonza, \& Guignon, 2014). In fact, a relevant parameter is certainly the existence of hydration-water clusters that can promote the LDW-to-HDW transformation at a lower pressure. Hydration water is generally described as a dense layer of water molecules around solutes and its structure certainly resembles HDW. Such HDWlike clusters could act as nuclei able to trigger the transformation. As for other nucleation phenomena, the critical size of nuclei must be achieved for the transformation to happen. In the case of surfactant micelles, the transformation pressure was shifted to lower pressures when the surface of interaction between micelles and water was higher (Hidalgo Baltasar et al., 2014). In milk and caseinate solution samples which contain protein aggregates, such nucleation conditions were likely not met below $275 \mathrm{MPa}$. Moreover, since the LDW-HDW transformation in pure water, NaCas and skimmed milk are always observed around (275 \pm 5 ) MPa at room temperature, this means that the change in the surface of interaction between solutes (aggregates) and water is not very large from a system to another. The observed changes can be therefore mainly attributed to the effect of pressure on the characteristics of the hydrogen bonding network of water, regardless of the solute present in the aqueous solution.

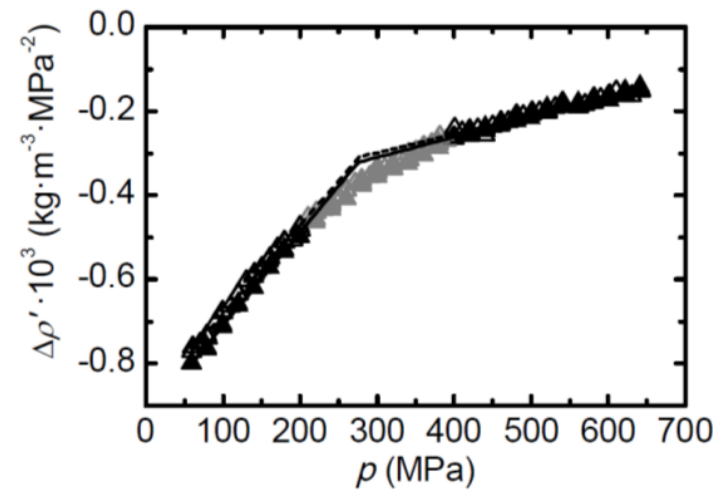

Figure 3. Numerical pressure derivatives of $(\partial \rho / \partial p)_{s}$ for sodium caseinate solution $(\boldsymbol{\Delta})$ and skimmed milk $(\Delta)$ (data are almost superimposed). The two lines are the results of fittings to Equation (5) in each case. Grey points deviate from the limiting linear behavior due to the changes in HDW and LDW fractions; so they were disregarded from the fit. 


\section{Conclusions}

Speed-of-sound measurements in-situ under pressure have allowed standing out the structural transformation in pure water from HDW to LDW structure around 275 MPa at room temperature. This phenomenon was also detected in sodium caseinate solution and in skimmed milk at the same pressure. Therefore, milk components have no impact on the LDW-to-HDW transformation, at least up to $8 \% \mathrm{w} / \mathrm{v}$ content. This suggests that, when solutes are insensitive to pressure effects, any observed change (in any measured magnitude) around $275 \mathrm{MPa}$ is attributable exclusively to water, but if a change is observed at another pressure, solutes must have interfered with the transformation. When solutes are sensitive to pressure, this also suggests possible cooperative effects with other phenomena where water is involved such as gel-like phase formation around this pressure, as already found in surfactant model systems. A systematic study involving different kinds of solutes would be necessary to clarify the influence of solutes on the LDW-to-HDW transformation pressure.

The importance of pressure effect on water structure should not be overlooked when analyzing the mechanisms of food components interactions under pressure, especially in the $275 \mathrm{MPa}$ region at room temperature.

\section{Acknowledgements}

This work has been funded by the Spanish Ministry of Science and Innovation under project MALTA-Consolider Ingenio 2010 (CSD2007-00045), by the Spanish Ministry of Economy and Competitiveness under projects AGL2012-39756-C02-01/ALI, CTQ2015-67755-C2-1-R and MAT2015-71070-REDC, and by GRUPOS UCM 910481.

\section{References}

Baonza, V. G., Rull, F., \& Dubessy, J. (2012). Raman spectroscopy of gases, water and other geological fluids. In J. Dubessy, M.-C. Caumon, \& F. Rull (Eds), EMU Notes in Mineralogy (vol. 12, Chapter 8, pp. 277-318). London: European Mineralogical Union and the Mineralogical Society of Great Britain \& Ireland.

Devi, A. F., Buckow, R., Hemar, Y., \& Kasapis, S. (2013). Structuring dairy systems through high pressure processing. Journal of Food Engineering, 114 (1), 106-122.

Fanetti, S., Lapini, A., Pagliai, M., Citroni, M., Di Donato, M., Scandolo, S., Righini, R., \& Bini, R. (2014). Structure and dynamics of low-density and high-density liquid water at high pressure. Journal of Physical Chemistry Letters, 5 (1), 235-240.

Gebhardt, R., Takeda, N., Kulozik, U., \& Doster, W. (2011). Structure and stabilizing interactions of casein micelles probed by high-pressure light scattering and FTIR. Journal of Physical Chemistry B, 115 (10), 2349-2359.

Guignon, B., \& Baonza, V. G. (2016) Physical properties of fluid media. In J. Manuel Recio, J. Manuel Menéndez, \& A. Otero-de-la-Roza (Eds), An introduction to HighPressure Science and Technology (pp. 301-332). Boca Raton:CRC Press.

Harte, F. M., Gurram, S. R., Luedecke, L. O., Swanson, B. G., \& Barbosa-Cánovas, G. V. (2007). Effect of high hydrostatic pressure and whey proteins on the disruption of casein micelle isolates. Journal of Dairy Research, 74 (4), 452-458.

Hidalgo Baltasar, E., Taravillo, M., Baonza, V. G., Sanz, P. D., \& Guignon, B. (2011). Speed of sound in liquid water from (253.15 to 348.15) $\mathrm{K}$ and pressures 
from (0.1 to 700) MPa. Journal of Chemical and Engineering Data, 56 (12), 48004807.

Hidalgo Baltasar, E., Taravillo, M., Sanz, P. D., Baonza, V. G., \& Guignon, B. (2014). Role of water structure on the high pressure micellization and phase transformations of sodium dodecanoate aqueous solutions. Langmuir, 30 (25), 7343-7352.

Huppertz, T., \& De Kruif, C. G. (2006). Disruption and reassociation of casein micelles under high pressure: Influence of milk serum composition and casein micelle concentration. Journal of Agricultural and Food Chemistry, 54 (16), 59035909.

Koga, Y., Westh, P., Yoshida, K., Inaba, A., \& Nakazawa, Y. (2014). Gradual crossover in molecular organization of stable liquid $\mathrm{H}_{2} \mathrm{O}$ at moderately high pressure and temperature. A I P Advances, 4. doi:10.1063/1.4895536

Li, F., Cui, Q., He, Z., Cui, T., Zhang, J., Zhou, Q., Zou, G., \& Sasaki, S. (2005). High pressure-temperature Brillouin study of liquid water: Evidence of the structural transition from low-density water to high-density water. Journal of Chemical Physics, 123 (17), 174511.

Marco Saitta, A., \& Datchi, F. (2003). Structure and phase diagram of high-density water: The role of interstitial molecules. Physical Review E - Statistical, Nonlinear, and Soft Matter Physics, 67 (2 1), 020201, 202011-202014.

Moreno, F. J., Villamiel, M., \& Olano, A. (2003). Effect of high pressure on isomerization and degradation of lactose in alkaline media. Journal of Agricultural and Food Chemistry, 51 (7), 1894-1896.

Okhulkov, A. V., Demianets, Yu. N., \& Gorbaty, Yu. E. (1994). X-ray scattering in liquid water at pressures of up to $7.7 \mathrm{kbar}$ : Test of a fluctuation model. The Journal of Chemical Physics, 100 (2), 1578-1588.

Soper, A. K., \& Ricci, M. A. (2000). Structures of high-density and low-density water. Physical Review Letters, 84 (13), 2881.

Tromp, R. H., Huppertz, T., \& Kohlbrecher, J. (2014). Casein micelles at nonambient pressure studied by neutron scattering. Food Biophysics, 10 (1), 51-56.

Wagner, W., \& Pruss, A. (2002). The IAPWS formulation 1995 for the thermodynamic properties of ordinary water substance for general and scientific use. Journal of Physical and Chemical Reference Data, 31 (2), 387-535.

Weck, G., Eggert, J., Loubeyre, P., Desbiens, N., Bourasseau, E., Maillet, J.-B., Mezouar, M., \& Hanfland, M. (2009). Phase diagrams and isotopic effects of normal and deuterated water studied via X-ray diffraction up to $4.5 \mathrm{GPa}$ and $500 \mathrm{~K}$.

Physical Review B, 80, 180202(R). 